\title{
Comparing The \\ Usefulness Of Video And \\ Map Information In \\ Navigation Tasks
}

\section{Human-Robot Interaction}

\author{
Curtis W. Nielsen \\ Michael A. Goodrich
}

U.S. Department of Energy

National Laboratory

operated by

Battelle Energy Alliance

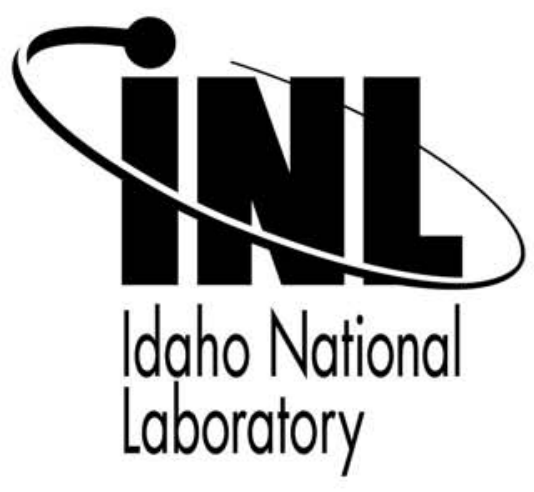

This is a preprint of a paper intended for publication in a journal or proceedings. Since changes may not be made before publication, this preprint should not be cited or reproduced without permission of the author. This document was prepared as an account of work sponsored by an agency of the United States Government. Neither the United States Government nor any agency thereof, or any of their employees, makes any warranty, expressed or implied, or assumes any legal liability or responsibility for any third party's use, or the results of such use, of any information, apparatus, product or process disclosed in this report, or represents that its use by such third party would not infringe privately owned rights. The views expressed in this paper are not necessarily those of the United States Government or the sponsoring agency. 


\section{Comparing the Usefulness of Video and Map Information in Navigation Tasks}

\author{
Curtis W. Nielsen \\ Brigham Young University \\ $3361 \mathrm{TMCB}$ \\ Provo, UT 84601 \\ curtisn@gmail.com
}

\author{
Michael A. Goodrich \\ Brigham Young University \\ $3361 \mathrm{TMCB}$ \\ Provo, UT 84601 \\ mike@cs.byu.edu
}

\begin{abstract}
One of the fundamental aspects of robot teleoperation is the ability to successfully navigate a robot through an environment. We define successful navigation to mean that the robot minimizes collisions and arrives at the destination in a timely manner. Often video and map information is presented to a robot operator to aid in navigation tasks. This paper addresses the usefulness of map and video information in a navigation task by comparing a side-by-side (2D) representation and an integrated (3D) representation in both a simulated and a real world study. The results suggest that sometimes video is more helpful than a map and other times a map is more helpful than video. From a design perspective, an integrated representation seems to help navigation more than placing map and video side-by-side.
\end{abstract}

\section{Categories and Subject Descriptors}

H.1.2 [Models and Principles]: User/Machine SystemsHuman factors, Human information processing

\section{General Terms}

Design, Experimentation, Human factors, Performance

\section{Keywords}

HRI, Human Robot Interaction, Information Presentation, Integrated Display, User Studies

\section{INTRODUCTION}

One of the fundamental aspects of robot teleoperation is the ability to successfully navigate a robot through an environment. We define successful navigation to mean that the robot minimizes collisions with obstacles and arrives at a destination in a timely manner. In order to support an operator in navigational tasks it is important to present navigation-relevant information to the operator. In remote, mobile robot navigation, it is common to use video and/or range information to inform the operator of obstacles and available directions of travel $[1,3,6,7,19]$.

Both video and range information provide distinct sets of information that have advantages and disadvantages for navigation tasks. For example, a video stream provides a visually rich set of information for interpreting the environment and comprehending obstacles, but it is usually limited by a narrow field of view and it is often difficult to comprehend how the robot's position and orientation relate to the environment. In contrast, range information is typically generated from infra-red sensors, laser range finders, or sonar sensors which detect distances and directions to obstacles, but do not provide more general knowledge about the environment. Advancements in map-building algorithms allow the integration of multiple range scans into maps which help an operator visualize how the robot's position and orientation relate to the environment.

In previous studies we used both video and range information (current readings or a map) to navigate a robot [13, 15]. During the experiments we observed that operators sometimes focused their attention on the map section of the interface and other times focused their attention on the section that contains the video.

These anecdotal observations lead to the question of how useful video and map information are for teleoperation. Although the ways to combine maps and visualization tools have been studied in other domains such as aviation (see, for example $[4,16]$ ) this problem has not been well studied in human-robot operation with occupancy grid maps.

This paper seeks to understand the usefulness of video and map information in navigation by comparing a prototypical $2 \mathrm{D}$ interface and a 3D augmented-virtuality interface [13, 15]. Specifically we hypothesized that for navigational tasks the video will hinder performance with the $2 \mathrm{D}$ interface, but minimally affect performance with the 3D interface. Further, we hypothesized that map information is more helpful to navigation than video information for both types of interface.

\section{MOTIVATION}

During the World Trade Center disaster in September 2001, Casper and Murphy used robots to search the rubble for victims [5]. Their robots were primarily operated via a video stream from a camera on the robot. One of their observations was that it was very difficult for an operator to handle both the navigation and the exploration of the environment with only video information.

In a separate study, Yanco and Drury had first responders 
search a mock environment using a robot that had camera, and map-building capabilities. One of their conclusions is that some participants considered the map useless because they felt it did not help them understand the robot's location [18]. Further, in an analysis of a robot competition, Yanco, Drury and Scholtz observed that many operators demonstrated a lack of awareness of the robot's location and surroundings [19].

Most mobile robot interfaces implement some aspect of video and/or range information to inform the operator of the environment around the robot. Some of these approaches present the information in a $2 \mathrm{D}$, side-by-side approach $[1$ $3,19]$ and others present the information integrated into a single 3D display $[12,7]$. In previous work an integrated display was found to be more useful for some navigation tasks in comparison to a side-by-side display [3, 13, 15].

To test the usefulness of map and video information in 2D and $3 \mathrm{D}$ interfaces, we next present two user studies: one in simulation and one using a real robot.

\section{EXPERIMENT 1}

In the first experiment we look at the usefulness of video and map information as aids for navigation with both a sideby-side approach (2D) and an integrated approach (3D). We hypothesized that with $2 \mathrm{D}$ interfaces video may negatively influence an operator's ability to perform a navigation task because it does not provide sufficient lateral information and it may draw the operator's attention away from more useful places on the interface such as a map or range information [9]. Furthermore, we hypothesized that with a $3 \mathrm{D}$ interface, video information will not hinder navigation when other range information is present. To explore the effect of range and video information on navigation, we assess an operator's ability to navigate a maze environment with two interfaces (2D and 3D) and three conditions for each interface (map-only, video-only, and map+video)

\subsection{Framework}

For this experiment we used a simulator based on the popular Unreal Tournament game engine as modified by Michael Lewis and colleagues at the University of Pittsburgh [11, 17]. Their modifications were originated with the intent of providing an inexpensive yet realistic simulator for studying urban search and rescue with mobile robots. The Unreal Tournament game engine provides a rich visual environment, which when combined with accurate models of common research robots and the game's physics engine provides for a very good mobile robot simulator [10].

We used the Unreal Tournament level editor to create maze environments that have the appearance of concrete bunkers which are filled with pipes, posters, windows, cabling, and electronic devices to provide a detailed environment for the robot to travel through. Some images of the virtual environment are shown in Figure 1.

The environment we created has seven separate mazes which are designed to explicitly test low-level navigation skills. There is only one path through each maze and no dead-ends, but it takes considerable teleoperation skill to navigate a maze from start to finish without crashing the robot. One of the mazes is used for training and the other 6 mazes are used for testing. The training maze contains a continuous path without an exit so that participants can practice driving the robot as long as desired.
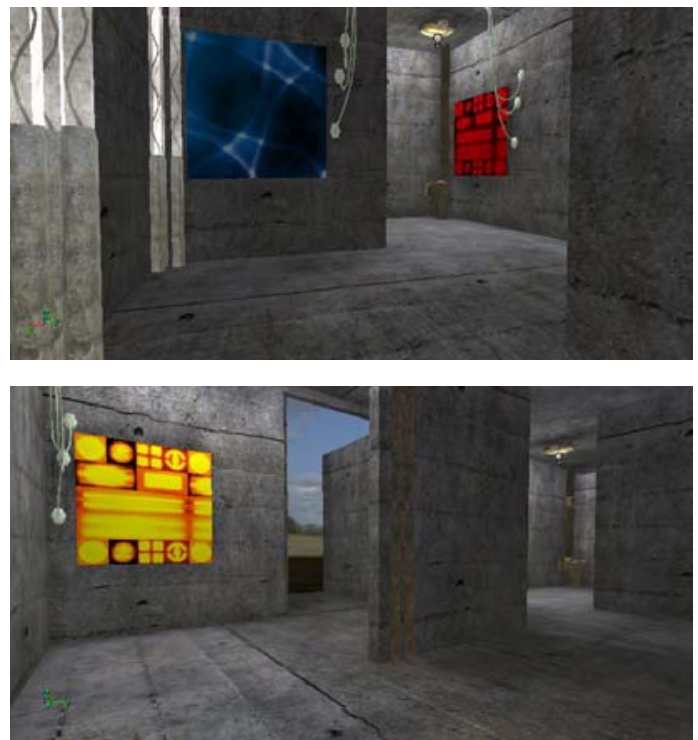

Figure 1: Images from the Unreal Tournament environment used for Experiment 1.

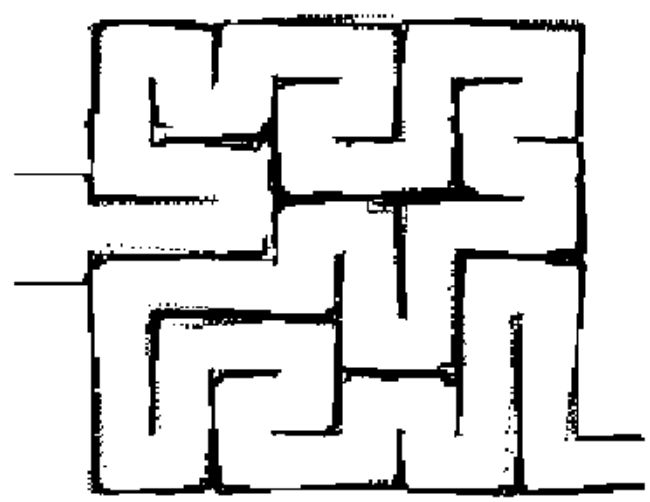

Figure 2: A map of one of the mazes used in Experiment 1 .

Each maze is an $8 \times 8$ grid where each cell in the grid is $2 \mathrm{x} 2$ meters for a total maze area of $256 \mathrm{~m}^{2}$. Each maze is designed to have 42 turns and 22 straight cells to minimize differences in results from different mazes (see Figure 2). The simulated robot used for this experiment is a model of the ATRV-Jr robot and has a width and length of 0.6 meters.

\subsubsection{Procedure}

Operators were instructed on how to drive the robot and how to perform the experiment through speakers on a headset, and they were told that their goal was to get the robot out of the maze as quickly as possible without hitting too many walls.

Before testing, operators were given a chance to practice driving the robot with both the $2 \mathrm{D}$ and the $3 \mathrm{D}$ interfaces. Each interface displayed both map and video information. The operators were asked to drive at least once through 


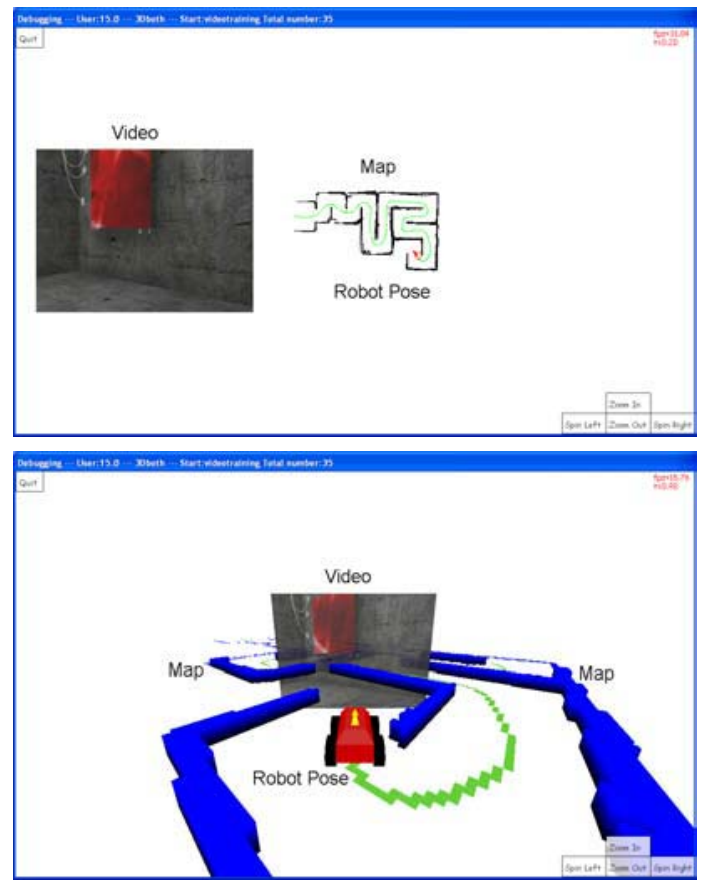

Figure 3: The 2D interface (top) and the 3D interface (bottom) used for Experiment 1.

the training maze to ensure a minimum amount of training. Once an operator had completed the training maze they were asked to continue practicing until they felt comfortable controlling the robot with the interface (most participants stopped training at this point). Following each training session and each experiment, participants were given a questionnaire to evaluate their performance. The purpose of the questionnaires after the training sessions was to familiarize the operators with the questions we would ask after each experiment.

Once training was complete, each participant was asked if they had any questions and they were told that the experiments would be very similar to the training, except that there would be an exit to the maze and that they would have different sets of information visible on the interface for each test. In particular, participants were given conditions of video-only, map-only, and map+video for both the 2D and $3 \mathrm{D}$ interfaces. For testing, we used a within-subjects counter-balanced design where each operator performed one test with each of the six conditions which were presented in a random order with the constraints that the 2D and 3D interfaces were used alternately and the conditions were counter balanced on which order they were used. The interfaces for the map+video conditions are shown in Figure 3.

\subsection{Results}

Twenty-four participants were paid to navigate a simulated robot with six different conditions of information presentation. Participants were recruited from the Brigham Young University community with most subjects enrolled as students. Two participants terminated the experiment prior to completion of the six conditions, but completed portions of the experiment were used for our analysis. Throughout

\begin{tabular}{|c|c|c|}
\hline & 2D Interface & 3D Interface \\
\hline Map-only & 258 & 196 \\
\hline Video-only & 366 & 351 \\
\hline \hline \% Change & $42 \%$ & $79 \%$ \\
\hline $\mathrm{p}$ & $7.8 e^{-4}$ & $1.6 e^{-7}$ \\
\hline
\end{tabular}

Table 1: Time to completion in Experiment 1.

\begin{tabular}{|c|c|c|}
\hline & 2D Interface & 3D Interface \\
\hline Map-only & 9.83 & 1.25 \\
\hline Video-only & 19.10 & 22.71 \\
\hline \hline \% Change & $94 \%$ & $18 \mathrm{x}$ \\
\hline $\mathrm{p}$ & $1.3 e^{-3}$ & $1.3 e^{-6}$ \\
\hline
\end{tabular}

Table 2: Number of collisions in Experiment 1.

the discussion of the results significance was obtained with a paired, two-tailed t-test with $n=24$ samples unless otherwise specified.

\subsubsection{Map-only vs. Video-only}

The results indicate that the video-only condition took significantly longer than the map-only condition for both the $2 \mathrm{D}(42 \%)$ and the $3 \mathrm{D}(79 \%)$ interfaces (see Table 1). Additionally, there were nearly twice as many collisions with the video-only condition in $2 \mathrm{D}$ than with the map-only condition and there were eighteen times the collisions with the $3 \mathrm{D}$ video-only condition in comparison to the $3 \mathrm{D}$ map-only condition (see Table 2 ). The $2 \mathrm{D}$ video-only and $3 \mathrm{D}$ videoonly conditions both had similar (not statistically different) results as measured by time to completion and the number of collisions. This is as we expected because the $3 \mathrm{D}$ and $2 \mathrm{D}$ interfaces present the video-only condition similarly.

\subsubsection{Map+video}

We found that with both the $2 \mathrm{D}$ and $3 \mathrm{D}$ interfaces, the map+video condition had results that were most similar to the map-only condition in comparison to the video-only condition (see Table 3). In particular we found that, on average, there were exactly the same number of collisions with the $3 \mathrm{D}$ interface for the map-only and map+video conditions and that there was no significant difference between the 2D map-only and map+video conditions. Figure 4 shows the average number of collisions for each of the six conditions.

On average there was an insignificant change in time to completion when video information was added to map information for both the $2 \mathrm{D}$ and $3 \mathrm{D}$ interfaces. However, we noticed a learning effect that took place with the $2 \mathrm{D}$ map-

\begin{tabular}{|c|c|c|}
\hline & $\begin{array}{c}\text { Time to Completion } \\
\text { (mean/stdev) }\end{array}$ & $\begin{array}{c}\text { Collisions } \\
\text { (mean/stdev })\end{array}$ \\
\hline 2D map-only & $258 / 57$ & $9.8 / 7.8$ \\
\hline 2D map+video & $271 / 55$ & $8.5 / 4.6$ \\
\hline 2D video-only & $366 / 118$ & $19.1 / 10.2$ \\
\hline \hline 3D map-only & $196 / 28$ & $1.3 / 2.2$ \\
\hline 3D map+video & $208 / 34$ & $1.3 / 1.8$ \\
\hline 3D video-only & $351 / 100$ & $22.7 / 14.4$ \\
\hline
\end{tabular}

Table 3: Comparison of the map+video condition to the map-only and video-only conditions in the simulation experiment. 


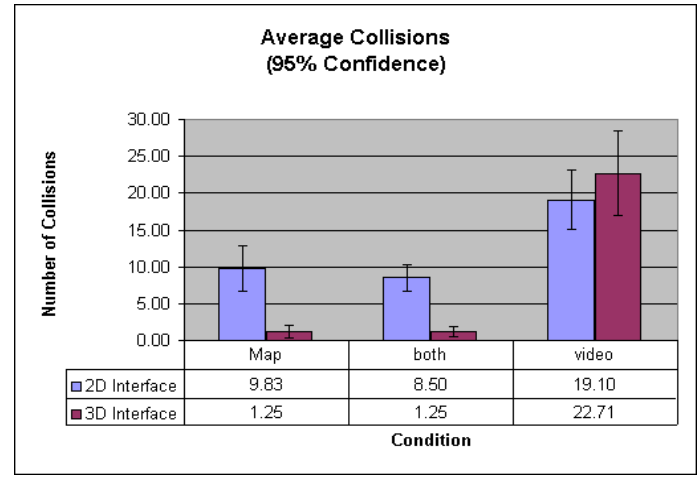

Figure 4: Number of collisions in Experiment 1.

\begin{tabular}{|c||c|c||c|c|}
\hline & First & Second & \% Change & $\mathrm{p}$ \\
\hline 2D map-only & 278 & 238 & $-14 \%$ & 0.0953 \\
\hline 2D map+video & 269 & 273 & $1.7 \%$ & 0.849 \\
\hline \hline \%change & $-3.1 \%$ & $15 \%$ & \multicolumn{2}{l}{} \\
\cline { 1 - 3 } $\mathrm{p}$ & 0.736 & 0.0909 & \multicolumn{2}{c}{} \\
\hline
\end{tabular}

Table 4: Time to completion in $2 \mathrm{D}$ after adjusting for learning.

only condition and the 3D map+video condition. In particular, the participants that used the 2D map-only condition after the 2D map+video condition finished the task $14 \%$ faster than the participants that used the 2D map-only condition before the $2 \mathrm{D}$ map+video condition $\left(\bar{x}_{2 D \text { map } 1}=278\right.$, $\bar{x}_{2 \text { map } 2}=238, p=.0953, n=12$, unpaired t-test, see Table 4).

Similarly, the participants that used the 3D map+video condition after the 3D map-only condition finished the task $15 \%$ faster than those that used the $3 \mathrm{D}$ map+video condition before the $3 \mathrm{D}$ map-only condition $\left(\bar{x}_{3 \text { Dmap }+ \text { video } 1}=\right.$ $225, \bar{x}_{3 \text { Dmap }+ \text { video } 2}=191, p=.0115, n=12$, unpaired t-test, see Table 5). We did not notice a learning effect between any of the other conditions.

When we compare the set of experiments in $2 \mathrm{D}$ where the map-only and map+video conditions were used first (Table 4), we find that adding video to the map has an insignificant effect. However in the set of experiments where the map-only and map+video conditions were used second, we find the time to completion of the task increases by $14.8 \%$ with the map+video condition in comparison to the map-only condition, which suggests that after accounting for learning, adding video to the map hurts navigation by increasing the time it takes an operator to navigate the robot out of a maze.

\begin{tabular}{|c||c|c||c|c|}
\hline & First & Second & \% Change & $\mathrm{p}$ \\
\hline 3D map-only & 195 & 196 & $0.32 \%$ & 0.961 \\
\hline 3D map+video & 225 & 191 & $-15 \%$ & 0.0115 \\
\hline \hline \% Change & $-15 \%$ & $-2.7 \%$ & \multicolumn{2}{|c}{} \\
\cline { 1 - 3 } $\mathrm{p}$ & 0.0357 & 0.626 & \multicolumn{2}{c}{} \\
\cline { 1 - 2 }
\end{tabular}

Table 5: Time to completion in $3 \mathrm{D}$ after adjusting for learning.

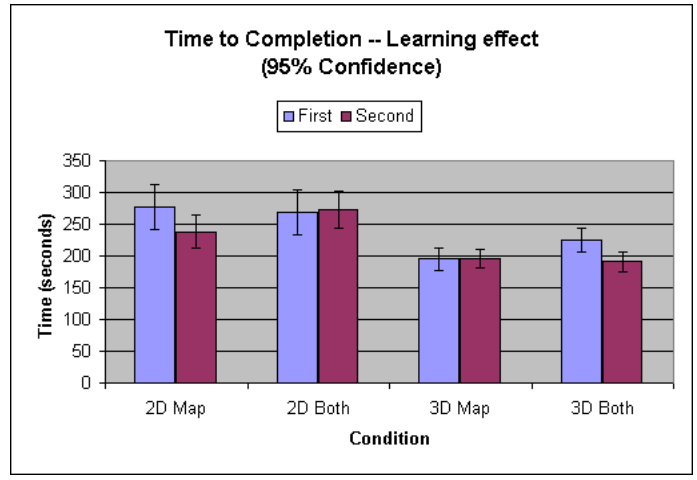

Figure 5: Time to completion after adjusting for learning in Experiment 1.

When we compare the set of experiments in $3 \mathrm{D}$ where the map-only and map+video conditions are used first (Table 5), we find that adding video to the map increases the time to completion by $15.2 \%$. However, in the set of experiments where the map-only and map+video conditions are used second, we find the difference in the time to complete the task is insignificant, which suggests that after accounting for learning, adding video to the map in the $3 \mathrm{D}$ interface does not affect the time it takes to navigate the robot out of the maze. A summary of the time to completion measurements when considering the learning effect is shown in Figure 5

\subsection{Discussion}

These results suggest that video can hurt navigation when the video does not contain sufficient navigational cues and video and map information are placed side-by-side. Even when map information is present and more useful than video for navigating, a novice operator's attention tends to be drawn towards the video, which, in this case, negatively affects their ability to navigate. These results make sense in light of research done by Kubey and Csikszentmihalyi which has shown that television draws attention because of the constantly changing visual scene [9]. It is interesting that even though it took longer to navigate, there were not more collisions with the 2D map+video condition than the 2D map-only condition, which implies that operators were not bumping into walls more, just moving slower through the maze.

\section{EXPERIMENT 2}

Experiment 1 provided an initial analysis of the usefulness of video and map information for performing navigation tasks with a remote, mobile robot in simulation. It is also useful to verify that the results and conclusions in simulation carry over and are applicable to environments and robots in the real world. For this purpose we have designed the second experiment to compare the usefulness of video and map information when navigating a robot in the real world. We hypothesized that the results would be similar to the results in simulation.

\subsection{Framework}

For this experiment we converted part of the second floor 

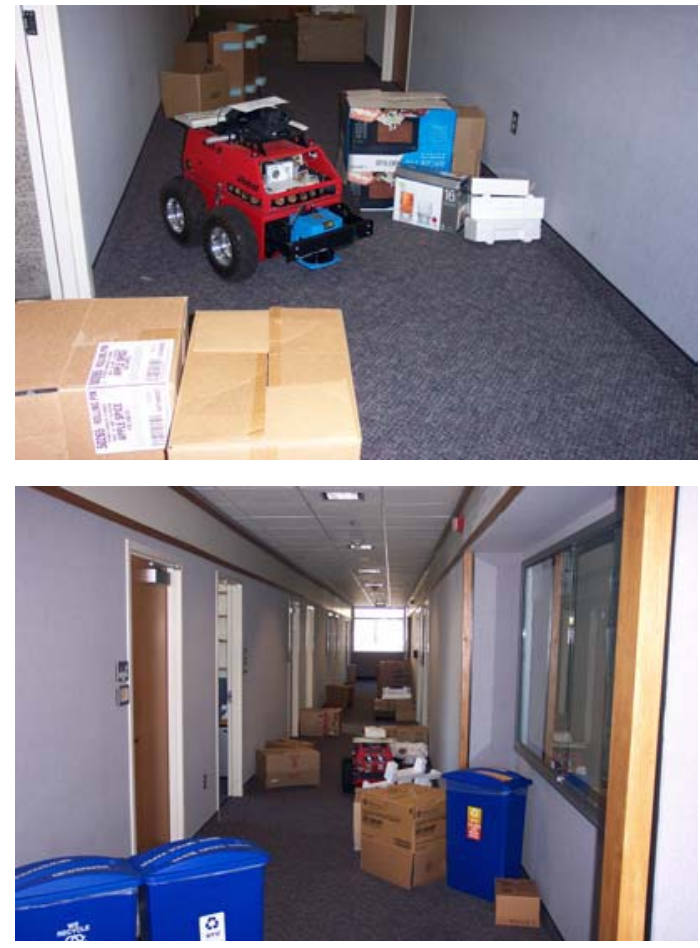

Figure 6: Images of the environment and the robot used for Experiment 2.

of the Computer Science Department at Brigham Young University into an obstacle course for our robot to travel through. The normal hallway width in the building is 2 meters and we used cardboard boxes, Styrofoam packing, and other obstacles to create a 50 meter course which has a minimum width of 1.2 meters. Figure 6 shows images of the robot and the two hallways used in the experiment.

\subsubsection{The Robot}

The robot used for the experiment is an ATRV-Jr which is approximately 0.6 meters in width and 0.7 meters in length (see Figure 6) . The robot uses artificial intelligence algorithms developed at the Idaho National Laboratory (INL) to safeguard it from colliding with walls and obstacles as it is teleoperated $[2,3]$. Additionally, the robot uses a mapbuilding algorithm developed by Konolige at the Stanford Research Institute (SRI) to represent the environment and localize the robot within the map [8].

The robot is controlled with a Microsoft Sidewinder 2 joystick ${ }^{1}$ and range and video information from the robot are presented to the operator via our 3D interface $[13,14]$. The 3D interface is integrated with the INL base station which handles the communication of movement commands and general information between the operator and the robot via radio modems. Live video from the robot is transmitted to the interface via $802.11 \mathrm{~b}$ wireless Ethernet. The interfaces used for this experiment have been modified from the previous experiment by including icons which indicate

${ }^{1}$ The INL base station did not support the steering wheel used in Experiment 1

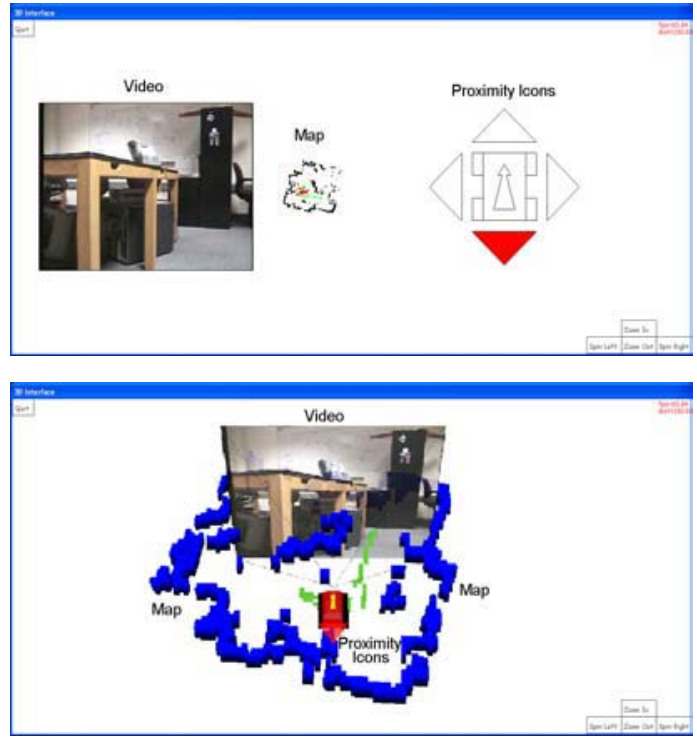

Figure 7: The 2D interface (top) and 3D interface (bottom) used for Experiment 2.

where the robot's intelligence identifies obstacles that might interfere with robot movement. The interfaces used for this experiment are shown in Figure 7.

\subsubsection{Procedure}

Before using the real robot, operators were trained to drive the robot with the Unreal Tournament training maze used in the first experiment. While training, operators drove the simulated robot with a joystick for a few minutes with each of the five conditions that they would be tested on $(2 \mathrm{D}$ map-only, 2D map+video, video-only ${ }^{2}$, 3D map-only, and 3D map+video). Upon completion of the training, the operators were moved to a different base station which was communicating with the real robot.

For testing, we used a within-subjects counter-balanced design where each operator used all five conditions in a pseudo-random order with the constraints that the $2 \mathrm{D}$ and 3D interfaces were used alternately and the conditions were counter-balanced on the order in which they were used. The experiment was setup such that an operator would drive the robot through the obstacle course with one condition, then at the end of the course an assistant would change the condition, turn the robot around, reset the map information, and start the next test. After every two runs the robot was plugged in for three to five minutes to keep the batteries charged.

\subsection{Results}

Twenty-one participants were paid to navigate the ATRVJr robot with five different conditions of information presentation. Participants were recruited from the Brigham Young University community with most subjects enrolled as students. The first three participants were used as part of a

${ }^{2}$ We did not compare $2 \mathrm{D}$ and $3 \mathrm{D}$ video-only conditions because in the previous experiment the video-only condition had similar results for both the 2D and 3D interfaces. 


\begin{tabular}{|c|c|c|}
\hline & 2D Interface & 3D Interface \\
\hline Map-only & 46.9 & 28.6 \\
\hline Video-only & 38.8 & 38.8 \\
\hline \hline \% Change & $-17 \%$ & $35 \%$ \\
\hline $\mathrm{p}$ & .663 & $2.4 e^{-2}$ \\
\hline
\end{tabular}

Table 6: Number of times the robot took initiative to protect itself in Experiment 2.

\begin{tabular}{|c|c|c|}
\hline & 2D Interface & 3D Interface \\
\hline Map-only & 319 & 227 \\
\hline Video-only & 243 & 243 \\
\hline \hline \% Change & $-24 \%$ & $7.2 \%$ \\
\hline $\mathrm{p}$ & $1.6 e^{-3}$ & .599 \\
\hline
\end{tabular}

Table 7: Time to completion in Experiment 2.

pilot study to determine a sufficient complexity of the obstacle course and to determine how best to use the robot while maintaining a sufficiently high charge on the batteries, therefore, there results were not included as part of the analysis. Additionally, the robot's responsiveness to commands was adversely affected by low batteries in eleven of the testing conditions (out of 90) therefore, this data was also discarded.

One of the differences between this experiment and the previous is that the real robot has intelligence on board to protect itself from hitting obstacles. For each test we recorded the number of times the robot acted to protect itself and discuss these results as robot initiative. Statistical significance was determined using a paired, two-tailed t-test with $n=18$ samples except as otherwise noted.

\subsubsection{Map-only vs. Video-only}

With the 3D interface, there was not a significant difference in the time to completion with the map-only and video-only conditions, however, the robot took initiative to protect itself nearly twice as much with the video-only condition than with the map-only condition $\left(\bar{x}_{\text {map }}=18.7\right.$, $\bar{x}_{\text {video }}=36.6, p=.0378$, see Table 6 ).

With the $2 \mathrm{D}$ interface, there was not a significant difference in the times the robot took initiative to protect itself with the map-only and video-only conditions, however, there was a significant difference in the time to complete the task. In fact, the results were opposite those from the simulated experiment. In particular it was $24 \%$ faster to use the video-only condition as opposed to the map-only condition $\left(\bar{x}_{\text {map }}=319 s, \bar{x}_{\text {video }}=243 s, p=1.6 e^{-3}\right.$, see Table 7$)$.

Most likely the reason these results differ from the previous experiment is that the environment in the second experiment provided more navigational cues visible in the video than the environment in the simulation experiment. In the simulation environment it was often the case that the video image was filled by a wall and none of the edges of the wall were visible. Moreover, the path through the simulation maze doubled back on itself numerous times, so the operator could not see very far in front of the robot. In contrast, for this second experiment, the edges of obstacles were nearly always visible through the camera and the operator could see future parts of the map as most obstacles were shorter than the height of the camera and there was only one 90 degree turn in the environment.

\begin{tabular}{|c|c|c|}
\hline & $\begin{array}{c}\text { Time to Completion } \\
\text { (mean/stdev) }\end{array}$ & $\begin{array}{c}\text { Robot Initiative } \\
\text { (mean/stdev) }\end{array}$ \\
\hline 2D map-only & $319 / 102$ & $46 . / 27.9$ \\
\hline 2D map+video & $247 / 54$ & $36.3 / 17.4$ \\
\hline \hline video-only & $243 / 59$ & $38.8 / 6.3$ \\
\hline \hline 3D map+video & $205 / 47$ & $24.8 / 13.5$ \\
\hline 3D map-only & $227 / 48$ & $28.6 / 20.1$ \\
\hline
\end{tabular}

Table 8: Comparison of the map+video condition to the map-only and video-only conditions in the real-world experiment.

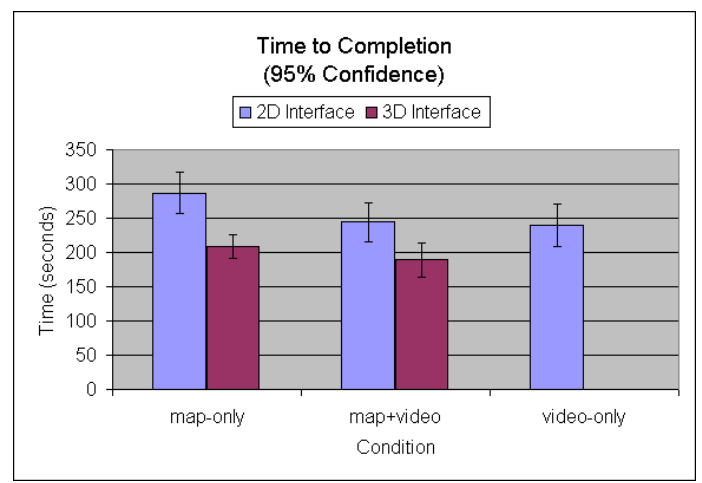

Figure 8: Time to completion for the five conditions in Experiment 2.

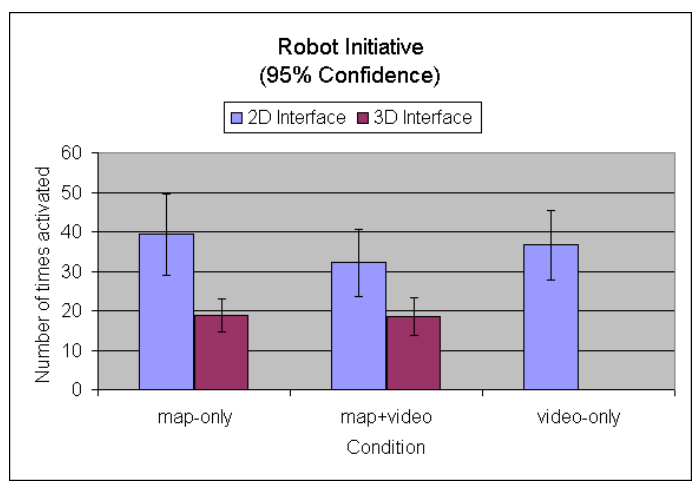

Figure 9: Number of times the robot took initiative to protect ltlself for the five conditions in Experiment 2.

\subsubsection{Map+video}

When map and video information were combined with the $2 \mathrm{D}$ interface, we found the results to be similar to the video-only condition with negligible difference in the time to completion and the number of collisions (see Table 8 and Figures 8 and 9).

When map and video information were combined with the $3 \mathrm{D}$ interface, the number of collisions are nearly identical to the map-only condition but we found that operators finished the obstacle course $9.6 \%$ faster with the map+video condition in comparison to the map-only condition $\left(\bar{x}_{\text {map }+ \text { video }}=\right.$ $205 s, \bar{x}_{v i d e o}=227 s, p=4.6 e^{-2}$, see Figure 8$)$.

This result is interesting because it suggests that when 
useful navigational information is available in both the map and the video, the 3D interface supports the complementary nature of the information and can lead to an improved performance over the individual parts. In contrast, performance with the $2 \mathrm{D}$ interface seems to be constrained by the best one can do with an individual set of information.

\subsection{Discussion}

To determine an ordering for the conditions, we define one condition to be better than another if both of the categories (time to completion and robot initative) are at least non-significantly different and one of the categories is significantly better. Conditions are considered equivalent if there is no statistical difference in either cateogry of analysis.

According to this criteria we found that when using the 3D interface, the map+video condition is better than the map-only condition (because the task took less time), and the map-only condition is better than the video-only condition (because there were fewer instances of robot initiative). These results suggest that when there is useful navigational information, in both the map and the video sets of information, integrating the information can yield better results than using either map or video individulally. Furthermore, when using the $2 \mathrm{D}$ interface, the map+video and the videoonly conditions are similar and are both better than the map-only condition (because the task took less time).

Interestingly, these results are different from our simulation studies where we found the video-only condition to be significantly worse than the other conditions. One complaint among participants with the $2 \mathrm{D}$ interface was that the map was too small (although it was the same relative size as the previous experiment) and that it was difficult to correlate the direction of the joystick movement with how the robot would move because the robot icon in the map was not always heading towards the top of the interface. Further, the map+video condition had results most similar to the video-only condition because the video tends to "pull" an operator's attention and hold it more than the map [9]. This assertion is further supported by the questions following the experiments, where operators claimed that most of their time was spent focused on the video.

\section{CONCLUSION}

Mobile robot navigation depends on the ability to see and comprehend information in the environment surrounding the robot. Typically information from the environment is presented to the operator via range and/or video, however, the manner in which this information is presented to an operator may affect navigational performance.

We have shown that video is helpful in environments where there are navigational cues in the video information, but video can diminish performance when there are minimal navigational cues. Furthermore, when video and map information are placed side-by-side they tend to compete for the operator's attention whereas when video and map information are integrated, they tend to complement each other and improve overall performance.

For design purposes, integrating maps with video in a 3D perspective seems much better than presenting map and video side-by-side in a $2 \mathrm{D}$ perspective. Most likely this is because the maps are always visible, even if the operator pays too much attention to the video. These results are consistent with previous results $[13,15]$.
In the future we plan to look at how delay affects navigation with both the 2D and 3D interfaces. Additionally we plan to look at exploration tasks using different interfaces and different sets of information.

\section{REFERENCES}

[1] M. Baker, R. Casey, B. Keyes, and H. A. Yanco. Improved interfaces for human-robot interaction in urban search and rescue. In Proceedings of the IEEE Conference on Systems, Man and Cybernetics, October 2004.

[2] D. J. Bruemmer, J. L. Marble, D. Dudenhoeffer, M. Anderson, and M. McKay. Mixed-initiative control for remote characterization of hazardous environments. In Proceedings of the Hawaii International Conference on System Sciences, Waikoloa, Hawaii, January 2003.

[3] D. J. Bruemmer, J. L. Marble, D. A. Few, R. L. Boring, M. C. Walton, and C. W. Nielsen. Let rover take over: A study of mixed-initiative control for remote robotic search and detection. IEEE Transactions on Systems, Man and Cybernetics-Part A: Systems and Humans, 35(4):494-504, July 2005.

[4] G. L. Calhoun, M. H. Draper, M. F. Abernathy, F. Delgado, and M. Patzek. Synthetic vision system for improving unmanned aerial vehicle operator situation awareness. In J. G. Verly, editor, Proceedings of SPIE Vol. 5802. p. 219-230, Enhanced and Synthetic Vision 2005. May 2005.

[5] J. Casper and R. R. Murphy. Human-robot interactions during the robot-assisted urban search and rescue response at the world trade center. IEEE Transactions on Systems, Man, and Cybernetics Part B, 33(3):367-385, June, 2003.

[6] T. W. Fong and C. Thorpe. Vehicle teleoperation interfaces Autonomous Robots, 11(1):9-18, July 2001.

[7] T. W. Fong, C. Thorpe, and C. Baur. Advanced interfaces for vehicle teleoperation: Collaborative control, sensor fusion displays, and remote driving tools. Autonomous Robots, 11(1):77-85, July 2001.

[8] K. Konolige. Large-scale map-making. In Proceedings of the National Conference on AI (AAAI), San Jose, CA, 2004.

[9] R. Kubey and M. Csikszentmihalyi. Television addiction is no mere metaphor. Scientific American, 286(2):62-68, 2002.

[10] M. Lewis and J. Jacobson. Game engines in research. Communications of the ACM, 45(1):27-48, 2002.

[11] M. Lewis, K. Sycara, and I. Nourbakhsh. Developing a testbed for studying human-robot interaction in urban search and rescue. In 10th International Conference on Human-Computer Interaction, Crete, Greece, 2003.

[12] R. Meier, T. Fong, C. Thorpe, and C. Baur. A sensor fusion based user interface for vehicle teleoperation. In International conference on field and service robotics (FSR), 1999.

[13] C. W. Nielsen, M. A. Goodrich, and R. J. Rupper. Towards facilitating the use of a pan-tilt camera on a mobile robot. In Proceedings of the 14th IEEE International Workshop on Robot and Human Interactive Communication (RO-MAN), Nashville, TN, 2005

[14] C. W. Nielsen, B. Ricks, M. A. Goodrich, D. J. Bruemmer, D. A. Few, and M. C. Walton. Snapshots for semantic maps. In Proceedings of the 2004 IEEE Conference on Systems, Man, and Cybernetics, The Hague, The Netherlands, 2004.

[15] B. W. Ricks, C. W. Nielsen, and M. A. Goodrich. Ecological displays for robot interaction: A new perspective. In International Conference on Intelligent Robots and Systems $I E E E / R S J$, Sendai, Japan, 2004.

[16] L. C. Thomas and C. D. Wickens. Eye-tracking and individual differences in off-normal event detection when flying with a synthetic vision system display. In Proceedings of the Human Factors and Ergonomics Society 48th Annual Meeting, Santa Monica, CA, 2004.

[17] J. Wang, M. Lewis, and J. Gennari. A game engine based simulation of the NIST urban search and rescue arenas. In Proceedings of the 2003 Winter Simulation Conference, 2003.

[18] H. A. Yanco and J. L. Drury. "Where am I?" Acquiring situation awareness using a remote robot platform. In Proceedings of the IEEE Conference on Systems, Man, and Cybernetics, October 2004.

[19] H. A. Yanco, J. L. Drury, and J. Scholtz. Beyond usability evaluation: Analysis of human-robot interaction at a major robotics competition. Journal of Human-Computer Interaction, 19(1 and 2):117-149, 2004. 